BULL. AUSTRAL. MATH. SOC.

\title{
CONDITIONAL EXPECTATION FOR OPERATOR-VALUED MEASURES AND FUNCTIONS
}

\author{
BRIAN JEFFERIES
}

A Radon-Nikodým theorem for operator-valued measures is applied to deduce the existence and uniqueness of conditional expectations in certain cases.

One of the central concepts of probability theory. is that of the conditional expectation of a real-valued random variable with respect to a probability measure. The possibility of treating perturbations of semigroups with operator-valued measures [5] suggests that an appropriate extension of the notion of conditional expectation to operator-valued measures and functions is in order.

Traditionally, the existence of conditional expectations is proved by appealing to the Radon-Nikodým theorem for scalar measures, and the proof transfers easily to Bochner integrable functions with values in a Banach space [1]. However, it is often inappropriate to assume that a function $f$ with values in the space $L(X)$ of continuous linear operators on a Banach space $X$ is Bochner integrable (with respect to the uniform operator topology). Usually $f$ will only be Pettis integrable in the space $L(X)$ equipped with the strong operator topology, a space which is metrizable only when $X$ is finite dimensional.

The purpose of this note is to use the variation of a vector measure

Received 13 June 1984.

Copyright Clearance Centre, Inc. Serial-fee code: 0004-9727/84 $\$$ A2.00 +0.00 . 
[4] to ensure the existence of conditional expectations in a certain class of measurable vector-valued functions, so that operator-valued functions may also be treated. For this purpose, the essential uniqueness of densities in this class [4] is crucial.

That the conditional expectation of a Pettis integrable function need not exist with values in the space in question [3] is related to the lack of completeness of the space of Pettis integrable functions in the semivariation topology. The Archimedes integral of Okada [7] is thereby relevant in this context.

The Radon-Nikodým theorems for operator-valued measures presented here are relevant to prediction and linear systems theory [2], and also to the Fubini theorem used in [5].

The terminology and notation of [4] is followed. Let $(\Omega, S, \lambda)$ be a finite measure space. Denote the completion of the $\sigma$-algebra $S$ with respect to $\lambda$ by $\bar{S}$. Let $E$ be a locally convex space.

A function $f: \Omega \rightarrow E$ is said to be $\lambda$-weakly regular if

(i) $f$ is $\sigma\left(E, E^{\prime}\right)$-Borel $\lambda$-measurable (that is, $f^{-1}(U) \in \bar{S}$ for every $\sigma\left(E, E^{\prime}\right)$-open set $\left.U \subset E\right)$, and

(ii) $\lambda \circ f^{-1}$ is a Radon measure on $E_{\sigma}$.

The following result is a consequence of [4] Corollary 4.

THEOREM 1. Let $m: S \rightarrow E$ be a vector measure such that $m<\lambda$. If $m$ has o-finite weakly compact variation, then $m$ has a $\lambda$-weakly regular density $f: \Omega \rightarrow E$ with respect to $\lambda$.

If $g: \Omega \rightarrow E$ is another $\lambda$-weakly regular density, then $f=g, \lambda$ almost everywhere.

If a $\lambda$-weakly regular density exists and $E$ is quasi-complete, then $m$ has $\sigma$-finite weakly compact variation [4]. The essential uniqueness for this class of densities is an important property, since it is wellknown that Pettis integrable densities are not unique in general [6, Example 6.1].

If $\lambda$ is a Radon measure, then every Lusin $\lambda$-measurable function is $\lambda$-weakly regular. The essential uniqueness for this class of densities was 
demonstrated by Thomas [9] for the case of vector-valued Radon measures. If $E$ is a Banach space, then the Dunford-Pettis-Phillips theorem implies that a function $f: \Omega \rightarrow E$ is $\lambda$-weakly regular if and only if it is strongly $\lambda$-measurable $[1]$.

Now let $F$ be a complete locally convex space. Then $L_{s}(E, F)$ denotes the space of continuous linear operators from $E$ into $F$ endowed with the topology of simple (pointwise) convergence. Let $F[\equiv F(E, F)]$ be the family of equicontinuous subsets $A$ of $L(E, F)$ such that for a dense set of $x \in E$, the set

$$
\{u x: u \in A\}
$$

is a relatively weakly compact subset of $F$.

THEOREM 2. Let $m: S \rightarrow L_{S}(E, F)$ be an operator-valued measure with $\sigma$-finite F-variation such that $m \ll \lambda$.

Then $m$ has a $\lambda$-weakly regular density $f: \Omega \rightarrow L_{s}(E, F)$.

Proof. According to Theorem 1, it suffices to prove that every $A \in F$ is relatively weakly compact in $L_{S}(E, F)$. Let $\bar{A}$ be the closure of $A \in F$ in $L_{s}\left(E, F_{\sigma}\right)$. From Schaefer [8], p. 139, $L_{s}(E, F)^{\prime}$ can be identified with $E \otimes F^{\prime}$, so $\bar{A}$ is the closure of $A$ in $\sigma\left(\mathrm{L}_{\mathrm{s}}(E, F), \mathrm{L}_{\mathrm{s}}(E, F) '\right)$.

Clearly $\bar{A} \in F$ and $\bar{A}$ is bounded in the weak topology of $L_{s}(E, F)$. By Tychonov's theorem, it suffices to show that $\bar{A}$ is closed in the weak completion of $L_{s}(E, E)$, which can be identified with the space $L\left(E, F^{\prime *}\right)$ of all linear maps from $E$ into the algebraic dual $F^{\prime *}$ of $F^{\prime}$ (choose a Hamel basis for $E$ ).

To prove this, let $G$ be a dense linear subspace of $E$ such that $\{u x: u \in \bar{A}\}$ is relatively weakly compact for each $x \in G$. Let $u_{\mathrm{K}} \in \bar{A}$, $\kappa \in K$ be a Cauchy net in $L_{s}\left(E, F_{\sigma}\right)$; that is, $\left\langle u_{\kappa} x, \xi\right\rangle, \kappa \in K$ is Cauchy for all $x \in E, \xi \in F^{\prime}$. For all $x \in G$, there exists $u x \in F$ such that $u_{K} x \rightarrow u x$ in $F_{\sigma}$, by virtue of the relative weak compactness of $\left\{u_{\kappa} x: \kappa \in K\right\}$. 
Furthermore, for every closed, disked neighbourhood $V$ of zero in $F$, there exists another, $U$ in $E$, such that $u_{K} U \subset V$ for all $\kappa \in K$. Thus, $u_{K}(U \cap G) \subset V, \kappa \in K$, and so $u(U \cap G) \subset V$, proving that $u: G \rightarrow F$ is the restriction of a linear map $\bar{u} \in L(E, F)$ such that $u_{K} \rightarrow \bar{u}$ in $L_{s}\left(E, F_{\sigma}\right)$. Consequently $A$ is a relatively weakly compact subset of $L_{s}(E, F)$.

If $A \in F$, then the proof above shows that for each $x \in E$, the set $C=\{u x: u \in A\}$ is relatively weakly compact, because $C$ is the image of the relatively weakly compact set $A$ under the continuous map $u \rightarrow u x$, $u \in A$. Nevertheless, it suffices to verify the condition only for a dense set of $x \in E$. If $E$ is barrelled, then the converse to Theorem 2 holds. INCw suppose that $(\Omega, S, \lambda)$ is a complete probability measure space. Let $T$ be a $\lambda$-complete sub- $\sigma$-algebra of $S$, and let $f: \Omega \rightarrow L(E, F)$ be a $\lambda$-integrable function in $L_{s}(E, F)$. If the restriction $f \lambda \mid T$ of the vector measure $f \lambda$ to the $\sigma$-algebra $T$ has $\sigma$-finite $F$-variation, then there exists an essentially unique $\lambda \mid T$-weakly regular function $\lambda(f \mid T): \Omega \rightarrow L_{s}(E, F)$ such that $\lambda(f \mid T)=f \lambda$ on $T$, by virtue of Theorem 2.

We call $\lambda(f \mid T)$ the weakly regular conditional expectation of $f$ with respect to $\lambda$. The essential uniqueness of the conditional expectation follows from Theorem 1 , and as a consequence of this essential uniqueness, the usual properties of conditional expectations (not involving order) hold. Without weak-regularity, it is possible to add a non-zero function with a vanishing indefinite integral to the conditional expectation [6].

It may happen that $f \lambda$ has $\sigma$-finite $F$-variation but $f \lambda \mid T$ does not [3]. Theorem $I$ also suggests other possibilities. Suppose that $f: \Omega \rightarrow E$ is a $\lambda$-integrable function, and $E$ densely embeds in the locally convex space $F$. Identify $E$ with its image as a subspace of $F$.

If $f \lambda \mid T$ has $\sigma$-finite weakly compact variation in $F$ (but not necessarily in $E$ ), then there exists an $F$-valued weakly regular conditional expectation $\lambda(f \mid T): \Omega+F$. For example, if $F$ is conuclear, this always holds. In the example of Heinich [3], we can take 
$E=l^{1}$ and $F=\left\langle l^{\infty}, \sigma\left(l^{\infty}, l^{l}\right)\right\rangle$.

The developments in [5] raise the following related question. Given an operator-valued measure $m: S \rightarrow L_{S}(E, F)$ and a scalar function $f: \Omega \rightarrow \mathbf{R}$ when does a scalar function $m(f \mid T): \Omega \rightarrow \mathbf{R}$ such that

$$
\int_{A} m(f \mid T) d m=\int_{A} f d m, A \in T,
$$

exist? For each $\xi \in L_{s}(E, F)^{\prime}$, we can find $(m, \xi)(f \mid T)$, but it may not be possible to knit the individual conditional expectations together into a scalar function. Thus, the question is that of differentiating a vector measure with respect to another vector measure, to obtain a scalar function.

The average range of a vector measure $m: S \rightarrow E$ with respect to $\lambda$ on $A \in S$ with $\lambda(A)>0$, is the set

$$
\mathrm{AR}_{A}(m, \lambda)=\{m(B) / \lambda(B): B \in S, B \subset A, \lambda(B)>0\}
$$

LEMMA 1. Let $E$ be a quasicomplete locally convex space. Let $f: \Omega \rightarrow E, g: \Omega \rightarrow E$ be $\lambda$-weakly regular $\lambda$-integrable functions, such that for all $A \in S$ with $\lambda(A)>0$, there exists $B \in S$ with $B \subset A$, $\lambda(B)>0$ such that for some number $K>0$,

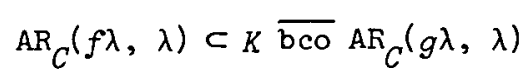

for every $C \in S$, with $C \subset B$ and $\lambda(C)>0$.

Then there exists a $\lambda$-measurable function $h: \Omega \rightarrow R$ such that $f(\omega)=h(\omega) g(\omega)$ for $\lambda$-almost all $\omega \in \Omega$.

Proof. By an exhaustion argument, it suffices to assume that the above condition holds for all $C \in S$ with $\lambda(C)>0$, and that $\operatorname{AR}_{\Omega}(g \lambda, \lambda)$ is a relatively weakly compact subset of $E$. Assume also that $S$ is $\lambda$-complete.

Let $\rho: L^{\infty}(\lambda)+L^{\infty}(\lambda)$ be a multiplicative lifting. According to [4], Corollary 4, it may be supposed that for all $\xi \in E^{\prime}$, $\rho(\xi \circ g)=\xi \circ g$ and $\rho(\xi \circ f)=\xi \circ f$, because any two $\lambda$-weakly regular densities must be equal almost everywhere. In particular, $f$ and $g$ are defined essentially independently of the choice of the lifting $\rho$. 
By assumption $\lambda \circ g^{-1}$ is a Radon measure on $E_{\sigma}$, so the support supp $\lambda \circ g^{-1}$ of $\lambda \circ g^{-1}$ is defined and has full measure. Let $\omega_{0} \in g^{-1}\left(\operatorname{supp} \lambda \circ g^{-1}\right)$. Then for every $\sigma\left(E, E^{\prime}\right)$-open neighbourhood $U$ of $g\left(\omega_{0}\right), \lambda\left(g^{-1}(U)\right)>0$.

Consequently,

$$
\cap{ }_{g^{-1}(U)}(f \lambda, \lambda) \subset K \quad \overline{\mathrm{bco}} \mathrm{AR} g^{-1}(U)(g \lambda, \lambda)
$$

where the intersection is over all basic open sets $U$ in $E_{\sigma}$ containing $g\left(\omega_{0}\right)$. Now, for each such $U$,

$$
{ }_{g^{-1}(U)}^{\mathrm{AR}}(g \lambda, \lambda) \subset \overline{\mathrm{bco}} g\left(g^{-1}(U)\right) \subset \overline{\mathrm{bco}} U
$$

by the Hahn-Banach theorem, so we have

$$
\cap_{g^{-1}(U)}(f \lambda, \lambda) \subset K \quad \overline{\mathrm{bco}} U
$$

Appealing to the Hahn-Banach theorem again,

$$
=\left\{a g\left(\omega_{0}\right):|a| \leq k\right\} \text {. }
$$

Fix a basic open set $U \subset E_{\sigma}$ containing $g\left(\omega_{0}\right)$ and put $\mu(A)=\lambda\left(A \cap g^{-1}(U)\right), A \in S$. Then $\mu \circ f^{-1}$ is a Radon measure on $E_{\sigma}$, and according to the proof of [4], Corollary 4, if $C$ denotes the $\sigma\left(E, E^{\prime}\right)$-closure of the average range of $f \lambda$ with respect to $\lambda$ on $g^{-1}(U)$, then $\mu \circ f^{-1}(C)=\lambda\left(g^{-1}(U)\right)$; that is, $f(\omega) \in C$ for $\lambda$-almost all $\omega \in g^{-1}(U)$.

Suppose that $\xi \in C^{\circ}$, the polar of $C$ in $E$. Then $|\langle f(\omega), \xi\rangle| \leq 1$ for $\lambda$-almost all $\omega \in g^{-1}(U)$. Put $A=g^{-1}(U)$. Then $x_{A}|\langle f, \xi\rangle| \leq 1$, $\lambda$-almost everywhere. The set $U$ is the finite intersection of open halfplanes, so that $\rho\left(x_{A}\right)=x_{A}, \rho(\langle f, \xi\rangle)=\langle f, \xi\rangle$ and $\rho\left(x_{A}\langle f, \xi\rangle\right)=\rho\left(x_{A}\right) \rho(\langle f, \xi\rangle)=x_{A}\langle f, \xi)$. Since $\rho$ is an isometry from $L^{\infty}(\lambda)$ to $L^{\infty}(\lambda), \chi_{A}|\langle f, \xi\rangle| \leq 1$ everywhere; that is, 


$$
f(\omega) \in \overline{\mathrm{bco}} C
$$

for all $\omega \in g^{-1}(U)$. Because $\omega_{0} \in \mathrm{ng}^{-1}(U)$, we have

$$
f\left(\omega_{0}\right) \in\left\{a g\left(\omega_{0}\right):|a| \leq K\right\}
$$

If $g\left(\omega_{0}\right)=0$, put $h\left(\omega_{0}\right)=0$ and $f\left(\omega_{0}\right)=h\left(\omega_{0}\right) g\left(\omega_{0}\right)$ otherwise, so defining $h$ on a set of full measure.

Let $m: S \rightarrow E, \quad n: S \rightarrow E$ be vector measures such that $m \ll \lambda$ and $n \ll \lambda$. We write $(m, \lambda) \ll(n, \lambda)$ if for all $A \in S$ with $\lambda(A)>0$ there exists $B \in S$ with $\lambda(B)>0, B \subset A$ such that for some number $K>0$,

$$
\operatorname{AR}_{C}(m, \lambda) \subset K \overline{\mathrm{bco} \mathrm{AR}_{C}(n, \lambda)}
$$

for all $C \in S$ such that $C \subset B$ and $\lambda(C)>0$.

THEOREM 3. Let $m: S \rightarrow E, n: S \rightarrow E$ be vector measures such that $(m, \lambda) \ll(n, \lambda)$. Suppose that $n$ has o-finite weakly compact variation.

Then there exists a $\lambda$-measurable fronction $h: \Omega \rightarrow \mathbb{R}$ such that $m=h n$.

Proof. By [4], Theorem 3, both $m$ and $n$ have o-finite weakly compact variation, so by Theorem 1 , there exist $\lambda$-weakly regular functions $f: \Omega \rightarrow E, g: \Omega \rightarrow E$ such that $m=f \lambda, n=g \lambda$. Thus Lemma 1 applies to give $h: \Omega \rightarrow \mathbb{R}$ (under the assumptions, quasicompleteness is unnecessary).

It is easily seen that if such a function $h$ exists, then we must have $(m, \lambda) \ll(n, \lambda)$. Returning to the case of conditional expectations, suppose that $m: S \rightarrow L_{S}(E, F)$ is an operator-valued measure. If $f: \Omega \rightarrow \mathbf{R}$ is an m-integrable function, then a conditional expectation $m(f \mid T): \Omega \rightarrow \mathbf{R}$ of $f$ with respect to $m$ exists whenever $m \mid T$ has $\sigma$-finite $F$-variation and

$$
(f m|T, \lambda| T) \ll(m|T, \lambda| T) .
$$

Another application of Theorem 2 is relevant to Fubini's theorem used in [5, Proposition 4]. Let $m: S \rightarrow L_{S}(E, F)$ be an operator-valued 
measure. Let $(\Sigma, E, \mu)$ be a finite measure space. Suppose that the function $f: \Omega \times \Sigma \rightarrow R$ is $m \otimes \mu$-integrable in $L_{s}(E, F)$. Then it is easy to show that for m-almost every $\omega \in \Omega$ the function $\sigma \rightarrow f(\omega, \sigma)$, $\sigma \in \Sigma$, is $\mu$-integrable and

$$
\int_{\Omega \times \Sigma} f d m \otimes \mu=\int_{\Omega}\left[\int_{\Sigma} f(\omega, \sigma) d \mu(\sigma)\right] d m(\omega)
$$

(see [5] for details). However, it need not be the case that for $\mu$-almost all $\sigma \in \Sigma$, the function $\omega \rightarrow f(\omega, \sigma), \omega \in \Omega$ is m-integrable in $L_{s}(E, F),[5]$.

Suppose that $F$ densely embeds in the complete locally convex space $G$. Then $L(E, F)$ can be identified with a subspace of $L(E, G)$. Define

$$
M(B)(A)=\int_{A \times B} f d m \otimes \mu, A \in S, B \in E .
$$

Then for each $B \in E, M(B)$ belongs to the space $X$ of $L_{s}(E, G)$-valued measures on $S$. Let $G$ be the family of sets $S \subset X$ such that

(i) for each $\xi \in L_{s}(E, G)^{\prime}$, the set of measures

$$
\{\langle n, \xi\rangle: n \in S\}
$$

is uniformly countably additive, and

(ii) for every $A \in S$, the set of operators

$$
\{n(A): n \in S\}
$$

belongs to the family $F(E, G)$.

Suppose that the vector measure $M$ has $\sigma$-finite $G$-variation. Then Theorem 2 ensures that for $\mu$-almost all $\sigma \in \Sigma$, the function $\omega \rightarrow f(\omega, \sigma), \omega \in \Omega$ is m-integrable in $L_{s}(E, G)$ and

$$
\int_{\Omega \times \Sigma} f d m \otimes \mu=\int_{\Sigma}\left[\int_{\Omega} f(\omega, \sigma) d m(\omega)\right] d \mu(\sigma) \text {. }
$$

An application of this result to the perturbations of semigroups is given in [5]. 


\section{References}

[1] J. Diestel and J.J. UhI, Vector measures (Mathematical Surveys, 15. American Mathematical Society, Providence, Rhode Island, 1977).

[2] W. Hackenbroch, "On the Radon-Nikodým theorem for operator measures and its applications to prediction and linear systems theory, 193-206 (Lecture Notes in Mathematics, 695. Springer-Verlag, Berlin, Heidelberg, New York, 1978).

[3] H. Heinich, "Esperance conditionelle pour les fonctions vectorielles", C.R. Acad. Sci. Paris Sér. A 276 (1973), 935-938.

[4] Brian Jefferies, "The variation of vector measures and cylindrical concentration", IZZinois J. Math. (to appear).

[5] I. KI uvánek, "Operator-valued measures and perturbations of semigroups", Arch. Rat. Mech. Anal. 81 (1983), 161-180.

[6] J. Kupka, "Radon-Nikodým theorems for vector valued measures", Trans. Amer. Math. Soc. 169 (1972), 197-217.

[7] S. Okada, "Integration of vector-valued functions", Proc. Measure Theory Conf. (Lecture Notes in Mathematics, 1033. SpringerVerlag, Berlin, Heidelberg, New York, 1982).

[8] H.H. Schaefer, Topological vector spaces (Springer-Verlag, New York, Heidelberg, Berlin, 1971).

[9] G.E.F. Thomas, The Lebesgue-Nikodym theorem for vector-valued Radon measures (Memoirs of the American Mathematical Society, 139. American Mathematical Society, Providence, Rhode Island, 1974).

Centre for Mathematical Analysis,

Australian National University,

GPO Box 4,

Canberra, ACT 2601,

Australia.

Present Address:

Department of Mathematics,

Ohio State University,

230 West 18th Avenue,

Col umbus, Ohio 43210 ,

USA. 